Sharif University of Technology
Scientia Iranica
Transactions E: Industrial Engineering
hCIENTIA

\title{
Applying a change-point control chart based on likelihood ratio to supply chain network monitoring
}

\author{
J. Zhong ${ }^{\mathrm{a}}$, X. Hu ${ }^{\mathrm{b}}, \mathrm{Y}$. Yang ${ }^{\mathrm{c}}$, and Y.L. Tu ${ }^{\mathrm{d}, *}$ \\ a. College of Management \& College of Tourism, Fujian Agriculture and Forestry University, Fuzhou, 350002, China. \\ b. School of Management, Nanjing University of Posts and Telecommunications, Nanjing, 210023, China. \\ c. Department of Management Science and Engineering, Nanjing University of Science and Technology, Nanjing, 210094, China. \\ d. Department of Mechanical and Manufacturing Engineering, University of Calgary, Calgary, T2N 1N4, Canada.
}

Received 21 November 2017; received in revised form 30 November 2018; accepted 21 July 2019

\section{KEYWORDS}

Supply chain network system;

Serial-parallel multistage process; Order completion

time;

State-space modeling; Change-point chart based on likelihood ratio.

\begin{abstract}
A supply chain network system was regarded as a serial-parallel multistage process and the application of a change-point control chart based on likelihood ratio was explored to monitor this system. First, state-space modeling was used to characterize complexities of the supply chain network system. Second, a change-point control chart based on likelihood ratio was used to trigger potential tardy orders in the system. Third, a case study was carried out to prove that the change-point control chart could effectively signal mean shift in completion time of one order in one stage and could accurately estimate the change point and the out-of-control stage in terms of performance indices. In detail, when the mean shift was relatively small, the change-point control chart could effectively identify it and more accurately detect the change point and the out-of-control stage than the traditional Shewhart control chart did. We also investigated the effect of misspecified parameters of state space equations on performance of the change-point control chart. The results showed that the performance of the change-point control chart could still remain relatively stable. In general, the change-point control could effectively monitor the supply chain network system and the monitoring effect was relatively stable.
\end{abstract}

(C) 2020 Sharif University of Technology. All rights reserved.

\section{Introduction}

A supply chain network system is full of uncertainties and variations, e.g., demand uncertainty, lead time variability, and supplier fault, which in turn cause degradation of the system performance [1,2]. If these variations and uncertainties accumulate up to a certain point, the supply chain network system may fail. A collapse of supply chain network system will significantly

*. Corresponding author. Tel.: +001 (403) 2204142 E-mail addresses: zhongjianlan_29@163.com (J.Zhong); hxl0419@hotmail.com (X.Hu);yang992942826@126.com (Y.Yang); paultu@ucalgary.ca (Y.L.Tu)

doi: $10.24200 /$ sci. 2019.5626 .1380 affect quality performance of the system [3]. Heydari [4] contended that the control of lead time variability was a motivation for persuading retailers to take part in cooperation programs. Especially in Make-To-Order (MTO) production mode, variability in response time is more significant. It would cause response time to go out of control. The response time is the interval from the time one customer places an order to the time the products/service is delivered to the customer. In the customized production environment, it is the order completion time. Due to many uncertainties in the order completion process, the response time must be monitored in real time in order to achieve a predetermined level of customer service.

Statistical Process Control (SPC) is a good technique for monitoring a supply chain network system. 


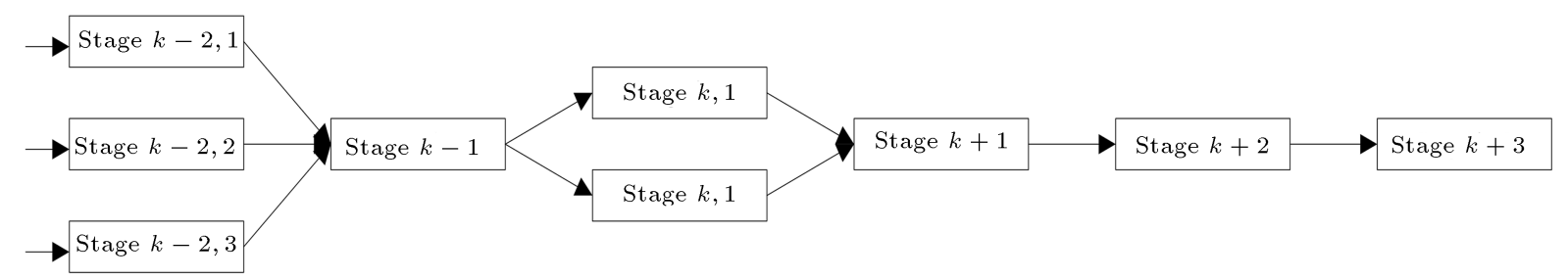

Figure 1. A supply chain network system viewed as a serial-parallel system.

As one of the tools, control charts can effectively detect assignable causes in the process to ensure stability of a system [5,6]. Wang [7] utilized the individual and moving range control charts on the basis of finite state machine theory to monitor order completion of each echelon (stage) in a supply chain network. An Exponentially Weighted Moving Average (EWMA) control chart was adopted by Chen et al. to detect variations in temperature of the cold chain [8]. Wang et al. [9] proposed a control chart using Kalman filtering to issue potential assignable events in a supply chain network. Faraz et al. [10] utilized a multivariate control chart to monitor delivery chain. Lu and Tsai [11] employed nonparametric generalized EWMA control chart in green supply chain management to constantly upgrade customer satisfaction of the stability of suppliers. Zhong et al. [12] used the control chart based on the maximum likelihood ratio to monitor supply chain systems and implemented the corresponding maintenance behaviors according to alarm signals.

In this study, a supply chain network system is put forward as a series or series-parallel multi-stage process (see Figure 1). Therefore, when using SPC to monitor this system, we must consider the correlation between stages [13]. Zolfaghari and Amiri [14] used a discriminant analysis control chart to monitor a twostage system with correlated variable-attribute quality characteristics. Furthermore, Amiri and Zolfaghari [15] extended this method to monitor a multi-stage system with clustering and indicated its effectiveness in estimating the change point. A change-point control chart can identify abnormal variation in the process and timely track process changes by measuring the difference between observation points [16]. Furthermore, the change-point control chart can estimate specific change points and the corresponding stages. The change-point control chart has widely been used in multistage processes (e.g., [17]). In addition, variation propagation is another important feature of the multistage process. Pirhooshyaran and Niaki [18] proposed a double-max multivariate EWMA control chart to jointly monitor the parameters of a multivariate multistage auto-correlated process with prior knowledge of variation propagation. Bazdar et al. [19] put forward a within-stage fault diagnosis approach on the basis of variation propagation modeling. Also, with respect to dynamic networks, Zou and Li [20] presented a Network
State Space Model (NSSM) to describe evolution of a dynamic network and integrated the NSSM with SPC for change detection.

In our study, we deal with a supply chain network system as a serial-parallel multistage process and explore the application of the change-point chart based on likelihood ratio to monitor the supply chain network. In detail, our monitoring object is the status (the time spent on various tasks or completion time) of each order at different stages in the supply chain network system. State-space model is used to characterize complexities of this system. Then, a change-point control chart based on likelihood ratio is used to trigger potential tardy orders in the system. Finally, a case study and a sensitivity analysis are provided to demonstrate effectiveness of the change-point control chart.

\section{Modeling of a supply chain network system}

A supply chain network system is a crossorganizational, cross-sectional composite system. Manufacturers, as the core business, may face multiple suppliers and distributors. The case that multiple suppliers provide parts or raw materials for one core business can be summarized a as many-to-one relationship and the case that one core business sales its products to multiple distributors or retailers can be summarized as a one-to-many relationship.

State-space modeling (see Eq. (1)) is used to quantitatively describe the system state of a supply chain network by transition equation as well as the relationship between measurement and the system state by measure equation.

$$
\begin{aligned}
& x_{k}=A_{k-1} X_{k-1}+U_{k}+\omega_{k}, \quad k=1,2, \ldots, N, \\
& y_{k}=C_{k} X_{k}+v_{k} .
\end{aligned}
$$

In fact, state space equations contain two types of quality information, namely state variables $x_{k}$ and measurement values $y_{k}$. These two types of quality information are present through all stages. We assume that the quality characteristics in this paper follow normal distribution. $A_{k-1}$ means how much quality information from stage $k-1$ is transferred to stage $k$ and $C_{k}$ represents the relationship between quality measurement $y_{k}$ and state vector $x_{k}$. Both $A_{k-1}$ 
and $C_{k}$ can be acquired by engineering knowledge and supply chain network information. $U_{k}$ denotes a process fault or an out-of-control condition, e.g., a tardy order. If the supply chain network system is incontrol, then $U_{k}=0$. In this paper, we assume that only step shifts of order completion time in one stage occur in the system, hence, $U_{k}$ is constant. $\omega_{k}$ denotes process noise, e.g., background disturbance and unmodeled errors. Finally, $v_{k}$ denotes measurement error.

For a specific order, the order completion time is the sum of the times spent on various tasks. Let $x_{i, k}$ be the state variable, which denotes the time that for order $i(i=1,2, \ldots)$ all the tasks are finished at stage $k(k=$ $1,2, \ldots, N)$, including the waiting time for the available resources before start of the processing task and the time spent on value-added processing task. We also assume the start time of the first task of the respective order equal to 0 , i.e., $x_{i, 0}=0$. When order $i(i=$ $1,2, \ldots)$ is completed at stage $k$, the time is measured and denoted by $y_{i, k}$.

As shown in Figure 1, a supply chain network can be decomposed into three scenarios. Scenario 1 plays out serial processes (i.e. from stage $k+1$ to stage $k+3$ ) in which both upstream and downstream stages have only one stream and the same propagation pattern. In Scenario 2 , the output of stage $k-1$ is separated at stage $k$; that is, the downstream stage has more than one workstation with the same input source. Scenario 3 merges the output of stage $k$ into stage $k+1$, i.e., the input has multiple sources while the output has only one stream.

According to [21], Scenario 1 (from stage $k+1$ to stage $k+3$ ) has a single propagation pattern. Therefore, Eq. (1) can be directly used in this case:

$$
\begin{aligned}
x_{i, k+2} & =A_{k+1} x_{i, k+1}+U_{i, k+2}+\omega_{k+2}, \\
i & =1,2, \ldots, \quad k=1,2, \ldots, N, \\
y_{i, k+2} & =C_{k+2} x_{i, k+2}+v_{k+2} .
\end{aligned}
$$

Hence, when the supply chain network system is incontrol, observation $y_{n}$ is maintained:

$$
y_{i, n}=\sum_{j=1}^{n} C_{n} \phi_{n, j} \omega_{j}+v_{n}
$$

where $\phi_{n, j}=A_{n-1} A_{n-2} \ldots A_{j}$. Therefore, $E\left(y_{i, n}\right)=0$, $\operatorname{Var}\left(y_{i, n}\right)=\sum_{i=1}^{n} C_{n}^{2} \phi_{n, j}^{2} \sigma_{\omega_{j}}^{2}+\sigma_{v_{n}}^{2}$.

Assume a mean shift occurs at stage $k+1$. Then, if $n<k$, observation $y_{n}$ holds; otherwise, i.e. $n \geq k$, it becomes:

$$
y_{i, n}=\sum_{j=1}^{n} C_{n} \phi_{n, j} \omega_{j}+v_{n}+C_{n} \phi_{n, k+1} U_{i, k+1} .
$$

Therefore:

$$
\begin{aligned}
& E\left(y_{i, n}\right)=C_{n} \phi_{n, k+1} U_{i, k+1} \\
& \operatorname{Var}\left(y_{i, n}\right)=\sum_{i=1}^{n} C_{n}^{2} \phi_{n, j}^{2} \sigma_{\omega_{j}}^{2}+\sigma_{v_{n}}^{2}
\end{aligned}
$$

In this case, once mean shifts occur at the upstream stage, only the means in the downstream stages are affected while their variances remain unchanged.

In Scenario 2 (from stage $k-1$ to stage $k$ in Figure 1), we have one stream at stage $k-1$ and two streams at stage $k$. Two dummy variables of quality characteristics, namely $\tilde{x}_{i, k-1,1}$ and $\tilde{x}_{i, k-1,2}$, are used to model this case, as shown in Eq. (5) [21]:

$$
\begin{aligned}
\left(\begin{array}{l}
x_{i, k, 1} \\
x_{i, k, 2}
\end{array}\right) & =A_{k-1} x_{i, k-1}+U_{i, k}+\omega_{k} \\
& =\left(\begin{array}{l}
A_{k-1} \tilde{x}_{i, k-1,1}+U_{i, k, 1}+\omega_{k, 1} \\
A_{k-1} \tilde{x}_{i, k-1,2}+U_{i, k, 2}+\omega_{k, 2}
\end{array}\right), \\
\left(\begin{array}{l}
y_{i, k, 1} \\
y_{i, k, 2}
\end{array}\right) & =\left(\begin{array}{l}
C_{k} x_{i, k, 1}+v_{k, 1} \\
C_{k} x_{i, k, 2}+v_{k, 2}
\end{array}\right),
\end{aligned}
$$

where $\tilde{x}_{i, k-1,1}$ and $\tilde{x}_{i, k-1,2}$ represent the virtual quality characteristics at stage $k-1$ transformed to the two workstations at stage $k$. We assume that the two workstations at stage $k$ are identical. Hence, $\tilde{x}_{i, k-1,1}$ and $\tilde{x}_{i, k-1,2}$ follow the same distribution and $x_{k-1}$ is their combination. Also, $\omega_{k, 1}$ and $\omega_{k, 2}$ follow the same distribution of $N\left(0, \sigma_{w_{k}}^{2}\right)$. Therefore, a mean shift that occurs at stage $k-1$ will affect both workstations at stage $k$ and the effect is the same. Thus, the propagation pattern of mean shifts in this case can be modeled as that in Scenario 1, i.e. the mean shifts but the variance remains unchanged.

In Scenario 3 (from stage $k$ to stage $k+1$ ), two workstations are at stage $k$. Hence, two dummy variables, namely $\tilde{x}_{i, k+1,1}$ and $\tilde{x}_{i, k+1,2}$, are used to model this case, as shown in Eq. (6):

$$
\begin{aligned}
x_{i, k+1} & =\left(\begin{array}{l}
\tilde{x}_{i, k+1,1} \\
\tilde{x}_{i, k+1,2}
\end{array}\right) \\
& =\left(\begin{array}{l}
A_{k} x_{i, k, 1}+U_{i, k+1,1}+\omega_{k+1,1} \\
A_{k} x_{i, k, 2}+U_{i, k+1,2}+\omega_{k+1,2}
\end{array}\right), \\
y_{i, k+1} & =C_{k+1} x_{i, k+1}+v_{k+1},
\end{aligned}
$$

where $\tilde{x}_{i, k+1,1}$ and $\tilde{x}_{i, k+1,2}$ denote the transformed quality characteristics from $x_{i, k, 1}$ and $x_{i, k, 2}$, respectively. $x_{i, k+1}$ is a combination of $\tilde{x}_{i, k+1,1}$ and $\tilde{x}_{i, k+1,2}$. If no mean shifts occur at the upstream stages, $\tilde{x}_{i, k+1,1}$ and $\tilde{x}_{i, k+1,2}$ will have the same distribution. Thus, the combined distribution of $x_{i, k+1}$ is the same as the distribution of $\tilde{x}_{i, k+1,1}$ and $\tilde{x}_{i, k+1,2}$.

We suppose that the portions of input to stage $k+$ 1 from workstation 1 and to stage $k$ from workstation 


$$
\begin{aligned}
& \mathrm{x}_{i, k+1}=\left(\begin{array}{l}
\tilde{x}_{i, k+1,1} \\
\tilde{x}_{i, k+1,2}
\end{array}\right)=\left(\begin{array}{l}
A_{k} A_{k-1} \tilde{x}_{i, k-1,1}+A_{k} U_{i, k, 1}+A_{k} \omega_{k, 1}+\omega_{k+1,1} \\
A_{k} A_{k-1} \tilde{x}_{i, k-1,2}+A_{k} U_{i, k, 2}+A_{k} \omega_{k, 2}+\omega_{k+1,2}
\end{array}\right), \\
& y_{i, k+1}=\left(\begin{array}{l}
\tilde{y}_{k+1,1} \\
\tilde{y}_{k+1,2}
\end{array}\right)=\left(\begin{array}{c}
C_{k+1} A_{k} A_{k-1} \tilde{x}_{i, k-1,1}+C_{k+1} A_{k} \omega_{k, 1}+C_{k+1} \omega_{k+1,1}+v_{k+1,1}+C_{k+1} A_{k} U_{i, k, 1} \\
C_{k+1} A_{k} A_{k-1} \tilde{x}_{i, k-1,2}+C_{k+1} A_{k} \omega_{k, 2}+C_{k+1} \omega_{k+1,2}+v_{k+1,2}+C_{k+1} A_{k} U_{i, k, 2}
\end{array}\right) .
\end{aligned}
$$

\section{Box I}

2 are $p_{k}$ and $1-p_{k}$, respectively. Combining Eqs. (5) and (6) (i.e. from stage $k-1$ to stage $k+1$ in Figure 1 ), the state space model of stage $k+1$ yields Eqs. (7) as shown in Box I.

On the basis of the combination of normal distribution, we have the mean $\left(\mu_{i, k+1,0}\right)$ and variance $\left(\sigma_{i, k+1,0}^{2}\right)$ of observation $y_{i, k+1}$ of order $i$ when the system is in-control:

$$
\begin{aligned}
& \mu_{i, k+1,0}=0 \\
& \sigma_{i, k+1,0}^{2}=\sum_{j=1}^{k+1} C_{k+1}^{2} \phi_{k+1, j}^{2} \sigma_{\omega_{i}}^{2}+\sigma_{v}^{2} .
\end{aligned}
$$

Also, the mean $\left(\mu_{i, k+1,1}\right)$ and variance $\left(\sigma_{i, k+1,1}^{2}\right)$ of observation $y_{i, k+1}$ of order $i$ when mean shifts of $U_{i, k, 1}$ and $U_{i, k, 2}$ occur are:

$$
\begin{aligned}
\mu_{i, k+1,1}= & p_{k} C_{k+1} A_{k} U_{i, k}, \\
\sigma_{i, k+1,1}^{2}= & \sum_{j=1}^{k+1} C_{k+1}^{2} \phi_{k+1, j}^{2} \sigma_{\omega_{i}}^{2}+\sigma_{v}^{2} \\
& +p_{k}\left(1-p_{k}\right)\left(C_{k+1} A_{k} U_{i, k}\right)^{2} .
\end{aligned}
$$

From Eq. (9), we can infer that the mean shift at the upstream stage impacts both means and variances of the downstream stages in scenario 3. Hence, a control chart, which is designed to monitor the supply chain network system, should be able to detect and distinguish mean shift and variance change, and identify variation source at the upstream stages or the current stage.

\section{A change-point control chart based on likelihood ratio}

A change-point control chart based on likelihood ratio is adopted from Sullivan and Woodall [22]. Suppose that sample size is $m$ and a change point $\tau$ occurs at the $\left(m_{1}+1\right)$ th sample point. Hence, the first $m_{1}$ samples follow normal distribution $N\left(\mu_{a}, \sigma_{a}^{2}\right)$ and the remaining samples $m_{2}\left(m_{2}=m-m_{1}\right)$ follow normal distribution $N\left(\mu_{b}, \sigma_{b}^{2}\right)$. The log-likelihood function for the $i$ th $(i=$ $1,2, \ldots \mathrm{m})$ sample can be expressed as:

$$
-\frac{1}{2} \log \left(2 \pi \sigma^{2}\right)-\frac{1}{2} \frac{\left(y_{i}-\mu\right)^{2}}{\sigma^{2}} .
$$

When the system is in-control, the log-likelihood function for population sample can be expressed as:

$$
l_{0}=-\frac{m}{2} \log (2 \pi)-\frac{m}{2} \log \sigma^{2}-\frac{m}{2},
$$

where $\bar{y}=\frac{\sum_{i=1}^{m} y_{i}}{m}$ and $\sigma^{2}=\frac{\sum_{i=1}^{m}\left(y_{i}-\bar{y}\right)^{2}}{m}$ denote the mean and variance of the maximum likelihood estimate for $m$ samples, respectively.

If a change point occurs, the log-likelihood function for the first $m_{1}$ samples can be expressed as:

$$
-\frac{m_{1}}{2} \log \left(2 \pi \sigma^{2}\right)-\frac{m_{1} \sigma_{a}^{2}}{2 \sigma^{2}}-\frac{m_{1}\left(\bar{y}_{1}-\mu\right)^{2}}{2 \sigma^{2}},
$$

where $\bar{y}_{1}=\frac{\sum_{i=1}^{m_{1}} y_{i}}{m_{1}}$ and $\sigma_{a}^{2}=\frac{\sum_{i=1}^{m_{1}}\left(y_{i}-\bar{y}_{1}\right)^{2}}{m_{1}}$ denote mean and variance of the maximum likelihood estimate for the first $m_{1}$ samples, respectively. Hence, the maximum log-likelihood function for the first $m_{1}$ samples is expressed as:

$$
l_{1}=-\frac{m_{1}}{2} \log (2 \pi)-\frac{m_{1}}{2} \log \sigma_{a}^{2}-\frac{m_{1}}{2} .
$$

Similarly, the maximum log-likelihood function for the remaining $m_{2}$ samples can be expressed as:

$$
l_{2}=-\frac{m_{2}}{2} \log (2 \pi)-\frac{m_{2}}{2} \log \sigma_{b}^{2}-\frac{m_{2}}{2},
$$

where $\bar{y}_{2}=\frac{\sum_{i=m_{1}+1}^{m} y_{i}}{m_{2}}$ and $\sigma_{b}^{2}=\frac{\sum_{i=m_{1}+1}^{m}\left(y_{i}-\bar{y}_{2}\right)^{2}}{m_{2}}$ denote mean and variance of the maximum likelihood estimate for $m_{2}$ samples, respectively.

Based on the test statistic for the abovementioned likelihood ratio, Sullivan and Woodall [22] proposed the following test statistic,

$$
\begin{aligned}
r t\left[m_{1}, m_{2}\right] & =-2\left[l_{0}-\left(l_{1}+l_{2}\right)\right] \\
& =m \log \left(\hat{\sigma}^{2}\left(\hat{\sigma}_{a}^{2}\right)^{-m_{1} / m}\left(\hat{\sigma}_{b}^{2}\right)^{-m_{2} / m}\right),
\end{aligned}
$$

when the test statistic is larger than a pre-specified critical value, we can obtain an estimator of $\tau$, i.e.

$$
\hat{\tau}=\underset{1 \leq m_{1}<m-1}{\arg } \max \left(\operatorname{lrt}\left[m_{1}, m_{2}\right]\right) .
$$

In order to explore whether the out-of-control state is caused by mean shift, variance change, or both, an 
estimator of process variance for $m$ samples can be rewritten as:

$$
\hat{\sigma}^{2}=\frac{m_{1} \hat{\sigma}_{a}^{2}+m_{2} \hat{\sigma}_{b}^{2}}{m}+\frac{m_{1} m_{2}}{m^{2}}\left(\bar{y}_{1}-\bar{y}_{2}\right)^{2} .
$$

Hence, the test statistic can be given as:

$$
\begin{aligned}
& \operatorname{lrt}\left[m_{1}, m_{2}\right]=M_{l r t}+V_{l r t}, \\
& \begin{aligned}
V_{l r t} & =m \log \left(\frac{m_{1}}{m} r^{2 m_{2} / m}+\frac{m_{2}}{m} r^{-2 m_{1} / m}\right) \\
& =m \log \left(\frac{1+c\left(r^{2}-1\right)}{r^{2 c}}\right), \\
M_{l r t} & =m \log \left(1+\frac{m_{1} m_{2}}{m\left(m_{1} \hat{\sigma}_{a}^{2}+m_{2} \hat{\sigma}_{b}^{2}\right)}\left(\bar{y}_{1}-\bar{y}_{2}\right)^{2}\right) \\
& =m \log \left(1+\frac{c(1-c)}{1+c\left(r^{2}-1\right)} d^{2}\right),
\end{aligned}
\end{aligned}
$$

where:

$$
c=\frac{m_{1}}{m}, \quad d=\frac{\bar{y}_{1}-\bar{y}_{2}}{\hat{\sigma}_{\mathrm{b}}}, \quad \text { and } \quad r=\frac{\hat{\sigma}_{a}}{\hat{\sigma}_{b}} .
$$

$M_{l r t}$ and $V_{l r t}$ represent mean shift and variance change, respectively. In Eq. (18), since $M_{l r t} \geq 0$, we achieve the minimum when the mean of the first $m_{1}$ samples equals that of $m_{2}$ samples and since $V_{l r t} \geq 0$, we achieve the minimum when the variance of the first $m_{1}$ samples equals that of $m_{2}$ samples.

The test statistic $\operatorname{lrt}\left[m_{1}, m_{2}\right]$ asymptotically follows gamma distribution $x^{2}(2)$ [22]. However, expectations of $\operatorname{lrt}\left[m_{1}, m_{2}\right]$ are different for different $m_{1}$. Hence, the test statistic is modified to make expectations of $\operatorname{lrt}\left[m_{1}, m_{2}\right]$ the same for different $m_{1}$.

$$
N \operatorname{lrt}\left[m_{1}, m_{2}\right]=\frac{\operatorname{lrt}\left[m_{1}, m_{2}\right]}{E\left(\operatorname{lrt}\left[m_{1}, m_{2}\right]\right)} .
$$

Then control limits (UCL) and expectation $E\left(\operatorname{lrt}\left[m_{1}\right.\right.$, $\left.m_{2}\right]$ ) can be obtained by the following simulation:

$$
\begin{aligned}
& U C L \approx \frac{1}{1.7} F^{-1}\left((1-\alpha)^{1 / k^{*}}\right), \\
& E\left(\operatorname{lrt}\left[m_{1}, m_{2}\right]\right) \approx 2\left(\frac{m_{1}+m_{2}-2}{\left(m_{1}-1\right)\left(m_{2}-1\right)}+1\right),
\end{aligned}
$$

where:

$$
k^{*}=-4.76+3.18 \log (\mathrm{m})
$$

$\alpha$ denotes probability of Type-I error and $F(\cdot)$ is cumulative distribution function of $\chi^{2}(2)$.

\section{Case study}

We take the production of computer server as an example. A server fulfillment supply chain network (as shown in Figure 2 [9]) is complicated, including customer, server fulfillment production, packing, shipping, etc. It is a typical series-parallel process. From the perspective of end customers, good service means on-time receipt of the server ordered. However, ontime delivery is not always met due to internal and external variations (e.g., machine downtime and loss of the quality of integrated circuits on the supplier side).

For simplicity, we focus on key elements of order fulfillment stages to verify the performance of the change-point control chart in detection of order tardiness. The average time spent on an order at each stage is shown in Table 1 when the system reaches a steady state. Standard deviation of the measurement error is 6 hours.

We consider only the case of probability of Type-I error when $\alpha=0.05$ and fix the number of observations

Table 1. Processing time at each stage (in hours).

\begin{tabular}{cccccccc}
\hline & $\boldsymbol{A}$ & $\boldsymbol{B 1} / \boldsymbol{B 2}$ & $\boldsymbol{C}$ & $\boldsymbol{D}$ & $\boldsymbol{E}$ & $\boldsymbol{F}$ & Total \\
\hline Mean & 10.33 & 55.00 & 48.33 & 56.67 & 12.67 & 40.00 & 223 \\
Std & 2.39 & 9.35 & 15.82 & 10.27 & 1.84 & 8.16 & \\
\hline
\end{tabular}

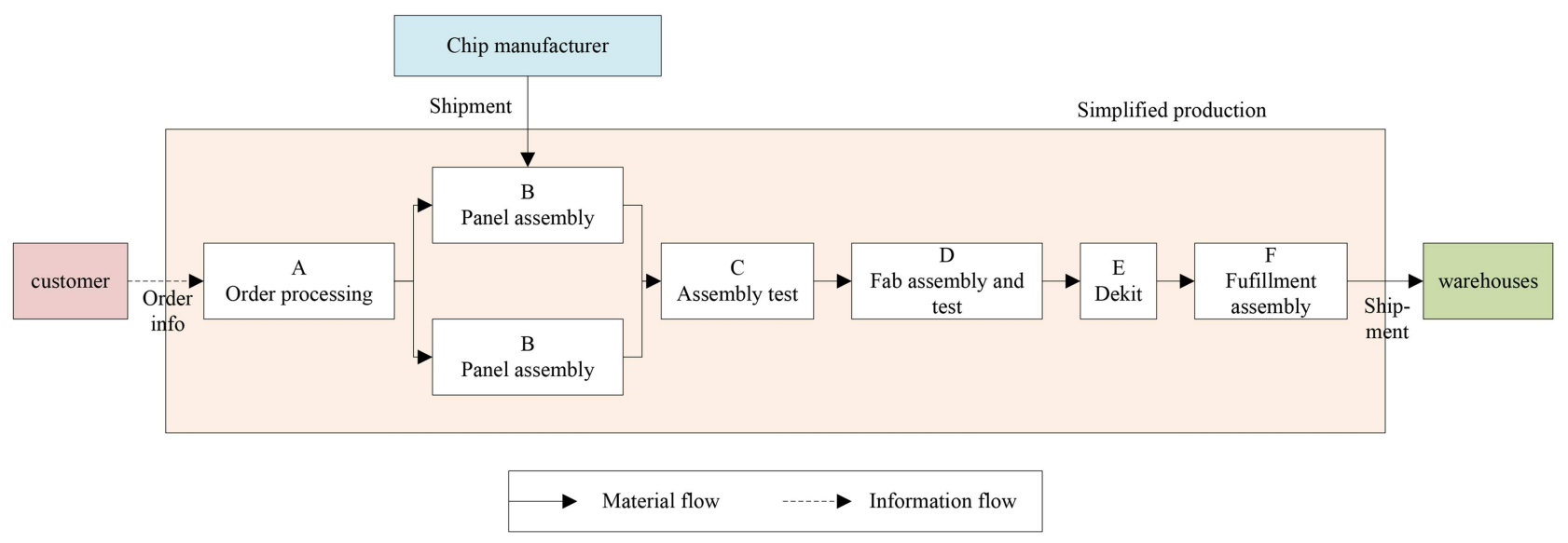

Figure 2. Six-stage serial-parallel process of simplified production. 
Table 2. Control limit of the traditional Shewhart control chart for each stage (in hours).

\begin{tabular}{ccccccc}
\hline & $\boldsymbol{A}$ & $\boldsymbol{B 1} \mathbf{1} \boldsymbol{B 2}$ & $\boldsymbol{C}$ & $\boldsymbol{D}$ & $\boldsymbol{E}$ & $\boldsymbol{F}$ \\
\hline UCL & 22.99 & 76.77 & 81.49 & 79.98 & 24.97 & 59.85 \\
\hline
\end{tabular}

Table 3. Control limit of the change-point control chart for each stage (in hours).

\begin{tabular}{ccccccc}
\hline & $\boldsymbol{A}$ & $\boldsymbol{B 1} / \boldsymbol{B 2}$ & $\boldsymbol{C}$ & $\boldsymbol{D}$ & $\boldsymbol{E}$ & $\boldsymbol{F}$ \\
\hline UCL & 4.07 & 3.02 & 4.07 & 4.07 & 4.07 & 4.07 \\
\hline
\end{tabular}

at $m=100$. Without loss of generality, we suppose $\left(C_{k}, A_{k}\right)=(1,1), \sigma_{w}^{2}=\sigma_{v}^{2}=1$, and $p_{B 1}=p_{B 2}=0.5$ according to engineering knowledge.

We first use the traditional Shewhart control chart to detect tardy orders at the end of each stage. Hence, $y_{k}$ is the variable for monitoring the system states $x$. Given the probability of Type-I error $\alpha=0.05$, the control limit of the Shewhart control chart can be calculated by:

$$
U C L_{s}(k)=E\left(\mathrm{y}_{k}\right)+r \sqrt{\operatorname{Var}_{y_{k}}}
$$

where $r$ denotes control limit parameters. Here, the control limit for each stage can be calculated by simulation, as shown in Table 2. If the order completion time exceeds the control limit, the control chart signals a true alarm. Thus, we can conclude that a tardy order has occurred at the current stage.

Now, we employ the change-point control chart based on the likelihood ratio introduced in Section 3 . The control limit for each stage can be calculated according to Eq. (20). The results are given in Table 3 .

We choose the following performance indices to judge the performance of the change-point control chart [23]: power of detection (the detection performance of the control chart when the order has been delayed, denoted by $p d$, for the change-point control chart), accuracy of estimation of the change point $\tau$ $\left(\mu_{\hat{\tau}}\right.$ and $\left.\sigma_{\hat{\tau}}\right)$ and initial out-of-control stage $(P(\hat{\xi}=\xi))$, and judgment about the mean shift or variance change $\left(M_{l r t}\right.$ and $\left.V_{l r t}\right)$. We also compare the two methods in terms of the power of detection (the power of detection for Shewhart control chart is denoted by $p d_{s}$ ).

As we known, detecting change points in a multistage process depends on the parameters $A_{k}$ and $C_{k}$, the initial out-of-control stage $\xi$, magnitude of the mean shifts in completion time at one stage $\delta$ (unexpected order delays), and the change point $\tau$. Hence, we consider the situation, as shown in Table 4, in which B1 and B2 denote two workstations at the parallel stage. Table 5 gives the performance for 10,000 replications by MATLAB.

Apparently, the change-point control chart is superior to the Shewhart control chart in detecting
Table 4. Combination of the values of $(\delta, \tau, \xi)$.

\begin{tabular}{cc}
\hline $\boldsymbol{\delta}$ & $(\boldsymbol{\tau}, \boldsymbol{\xi})$ \\
\hline 20 & $(30,2(B 1))(20,2(B 2))(40,3)$ \\
30 & $(30,2(B 1))(20,2(B 2))(40,3)$ \\
40 & $(30,2(B 1))(20,2(B 2))(40,3)$ \\
\hline
\end{tabular}

mean shift in terms of the power of detection. With the change-point control chart, estimation of the process change point is more accurate and the accuracy is gradually improved when mean shift increases in the cases of mean shifts at 20,30, and 40 (as shown in Column 4 of Table 5). We also considered other magnitudes of mean shift and found that the power of detection equaled 1 . The estimations of change point and initial out-of-control stage were close to the true values, especially with $\delta \geq 45$. Furthermore, considering the mean and standard deviation of the change point estimates (as shown in Columns 5 and 6 of Table 5), $\hat{\tau}$ performs uniformly better for any magnitude of mean shift. The probabilities $P(\hat{\xi}=\xi)$ also indicate that estimation of out-of-control stage is fairly accurate, especially for large mean shifts. In addition, as shown in the last two columns of Table 5, the values of $\bar{M}_{l r t}$ are much larger than the values of $\bar{V}_{l r t}$, which indicate that the system is out-ofcontrol because of the mean shifts in the completion time. In a word, the change-point control chart can identify the change point and the out-of-control stage, and distinguish the source of variation (mean shift or variance change).

\section{Sensitivity analysis}

The parameters $A_{k}$ and $C_{k}$ in state-space equations are usually estimated by sample data or derived by engineering knowledge. In practice, we may reach an inaccurate estimation of these parameters, especially for a large number of stages. Hence, we check the effectiveness and robustness of the change-point control chart in case of misspecification of the values of $A_{k}, C_{k}$.

We consider only $\alpha=0.05$ and $m=100$ and suppose that the true values of $A_{k}$ and $C_{k}$ are 1.0. Let $A_{k^{*}}$ and $C_{k^{*}}$ denote the incorrect values for stage $k^{*}$. We consider two situations: (1) $k^{*}=2$ and (2) $k^{*}=$ $1,3,5$. We also assume the change point at the serial stage $\xi=3, \tau=40$ and at the parallel stage $\xi=2$, $\tau=30$ (B1). For each situation, the following values of $\left(A_{k}, C_{k}\right)$ are considered (see Table 6$)$. Table 7 presents the power of detection, accuracy of estimation of the change point $\tau$ ( $\mu_{\hat{\tau}}$ and $\left.\sigma_{\hat{\tau}}\right)$, and judgment about mean or variance change $\left(M_{l r t}\right.$ and $\left.V_{l r t}\right)$, which is obtained by 10000 replications in MATLAB. The values in the fourth column of Table 7 are obtained when the values of $A_{k}$ and $C_{k}$ are pre-specified correctly. 
Table 5. Performance comparison.

\begin{tabular}{|c|c|c|c|c|c|c|c|c|}
\hline & \multirow{2}{*}{$\delta$} & \multirow{2}{*}{$\begin{array}{c}\text { Shewhart } \\
\qquad p d_{s}\end{array}$} & \multicolumn{6}{|c|}{ Change point } \\
\hline & & & $p d$ & $\mu_{\hat{\tau}}$ & $\sigma_{\hat{\tau}}$ & $P(\hat{\xi}=\xi)$ & $\bar{M}_{l r t}$ & $\bar{V}_{l r t}$ \\
\hline \multirow{3}{*}{$\begin{aligned} \tau & =30 \\
\xi & =2(B 1)\end{aligned}$} & 20 & 0.77 & 0.99 & 29.95 & 1.89 & 0.95 & 13.89 & 0.54 \\
\hline & 30 & 0.95 & 1.00 & 29.95 & 0.83 & 0.96 & 23.17 & 0.49 \\
\hline & 40 & 0.99 & 1.00 & 30.00 & 0.32 & 0.97 & 31.89 & 0.48 \\
\hline \multirow{3}{*}{$\begin{aligned} \tau & =20 \\
\xi & =2(B 2)\end{aligned}$} & 20 & 0.77 & 0.99 & 29.95 & 1.89 & 0.95 & 13.89 & 0.52 \\
\hline & 30 & 0.95 & 1.00 & 29.95 & 0.83 & 0.96 & 23.17 & 0.46 \\
\hline & 40 & 0.99 & 1.00 & 30.00 & 0.32 & 0.97 & 31.89 & 0.42 \\
\hline \multirow{3}{*}{$\begin{array}{l}\tau=40 \\
\xi=3\end{array}$} & 20 & 0.79 & 0.99 & 19.99 & 1.95 & 0.99 & 13.78 & 0.55 \\
\hline & 30 & 0.95 & 1.00 & 20.07 & 0.66 & 0.99 & 22.97 & 0.46 \\
\hline & 40 & 0.99 & 1.00 & 20.02 & 0.36 & 0.99 & 31.64 & 0.45 \\
\hline
\end{tabular}

Table 6. Combination of the values of $\left(C_{k^{*}}, A_{k^{*}}\right)$.

\begin{tabular}{cc}
\hline & $\left(C_{k^{*}}, A_{k^{*}}\right)$ \\
\hline $\mathrm{a}$ & $(0.8,1.0)$ \\
$\mathrm{b}$ & $(1.0,0.8)$ \\
$\mathrm{c}$ & $(0.8,0.8)$ \\
$\mathrm{d}$ & $(1.2,1.0)$ \\
$\mathrm{e}$ & $(1.0,1.2)$ \\
$\mathrm{f}$ & $(1.2,1.2)$ \\
$\mathrm{g}$ & $(1.2,0.8)$ \\
$\mathrm{h}$ & $(0.8,1.2)$ \\
\hline
\end{tabular}

For the case of serial sub-process, we can see that in situation (1) of underestimation, the change-point control chart still performs better except at the very small mean shifts, e.g. $\delta=20$. In this case, the power of detection changes slightly, ranging from 0.89 to 0.95 . Similarly, in situation (2), while many parameters are estimated incorrectly, the change-point control chart performs better except at very small mean shifts $\delta$ in terms of the power of detection.

For the case of the parallel sub-process, as shown in Table 8, the change-point control chart also performs better and even in the case of very small mean shifts, the power of detection remains almost unchanged.

The results show that the change-point control chart can still maintain a relatively stable monitoring effect when values of the parameters $A_{k}, C_{k}$ are misspecified. $\left(A_{k^{*}}, C_{k^{*}}\right)$ is a scalar greater than zero and the system is one-dimensional. Therefore, there are few changes in monitoring effect. In addition, the change-point control chart does not require any assumptions about process parameters. Moreover, the change-point control chart is applicable to processes with unknown process parameters and small numbers of in-control samples [23]. The sensitivity analysis verified this advantage. However, if $\left(A_{k^{*}}, C_{k^{*}}\right)$ is a high-dimensional matrix, the results may not be quite favorable. Of course, it is crucial to estimate $A_{k}$, and $C_{k}$ accurately when using a model-based method.

\section{Conclusion and suggestions for future research}

In this study, a supply chain network system was viewed as a serial-parallel multistage process and then, state-space technique was used to model this system. On this basis, we explored the application of the change-point control chart based on likelihood ratio under step shift in the mean of completion time at one stage. A case study was conducted to indicate that the change-point control chart could effectively signal process mean shifts and estimate the change point and the out-of-control stage. Finally, sensitivity analysis illustrated that there was no significant changes in the performance of the change-point control chart with misspecified parameters $A_{k^{*}}$ and $C_{k^{*}}$. In a word, the change-point control chart could effectively monitor the supply chain network system and the monitoring effect was relatively stable.

In this study, the case in which only one change point occurs in the supply chain network system has 
Table 7. Performance with misspecified values of the parameters $A_{k^{*}}$ and $C_{k^{*}}(\tau=40, \xi=3)$.

\begin{tabular}{|c|c|c|c|c|c|c|c|c|c|c|c|}
\hline$k^{*}$ & $\delta$ & Indicator & True & $a$ & $b$ & $c$ & $d$ & $e$ & $f$ & $g$ & $h$ \\
\hline \multirow[t]{15}{*}{2} & \multirow[t]{5}{*}{20} & $p d$ & 0.93 & 0.93 & 0.91 & 0.89 & 0.93 & 0.95 & 0.95 & 0.92 & 0.94 \\
\hline & & $\mu_{\hat{\tau}}$ & 40.27 & 40.19 & 40.01 & 40.45 & 40.23 & 40.45 & 39.96 & 40.26 & 40.36 \\
\hline & & $\sigma_{\hat{\tau}}$ & 4.00 & 4.02 & 5.38 & 5.21 & 4.01 & 4.66 & 3.99 & 4.99 & 4.37 \\
\hline & & $\bar{V}_{l r t}$ & 0.55 & 0.52 & 0.58 & 0.55 & 0.48 & 0.49 & 0.56 & 0.49 & 0.51 \\
\hline & & $\bar{M}_{l r t}$ & 14.30 & 14.49 & 13.50 & 13.18 & 14.30 & 14.90 & 14.36 & 13.82 & 14.86 \\
\hline & \multirow[t]{5}{*}{30} & $p d$ & 1.00 & 1.00 & 1.00 & 1.00 & 1.00 & 1.00 & 1.00 & 1.00 & 1.00 \\
\hline & & $\mu_{\hat{\tau}}$ & 40.08 & 40.16 & 40.05 & 39.99 & 40.06 & 40.07 & 40.10 & 40.05 & 40.00 \\
\hline & & $\sigma_{\hat{\tau}}$ & 1.67 & 1.76 & 2.11 & 2.08 & 1.81 & 1.67 & 1.67 & 1.87 & 1.69 \\
\hline & & $\bar{V}_{l r t}$ & 0.46 & 0.49 & 0.45 & 0.47 & 0.46 & 0.48 & 0.46 & 0.44 & 0.48 \\
\hline & & $\bar{M}_{l r t}$ & 25.39 & 25.20 & 24.26 & 23.37 & 25.51 & 26.13 & 25.69 & 24.26 & 26.37 \\
\hline & \multirow[t]{5}{*}{40} & $p d$ & 1.00 & 1.00 & 1.00 & 1.00 & 1.00 & 1.00 & 1.00 & 1.00 & 1.00 \\
\hline & & $\mu_{\hat{\tau}}$ & 39.94 & 39.99 & 40.01 & 40.04 & 39.93 & 40.01 & 39.96 & 40.02 & 40.04 \\
\hline & & $\sigma_{\hat{\tau}}$ & 0.95 & 0.89 & 0.96 & 1.15 & 0.95 & 0.89 & 0.94 & 0.99 & 0.92 \\
\hline & & $\bar{V}_{l r t}$ & 0.45 & 0.49 & 0.44 & 0.43 & 0.48 & 0.42 & 0.48 & 0.49 & 0.50 \\
\hline & & $\bar{M}_{l r t}$ & 38.19 & 37.92 & 36.01 & 35.24 & 37.67 & 38.87 & 37.85 & 36.23 & 39.18 \\
\hline \multirow[t]{15}{*}{$1,3,5$} & \multirow[t]{5}{*}{20} & $p d$ & 0.93 & 0.94 & 0.92 & 0.90 & 0.93 & 0.95 & 0.95 & 0.91 & 0.95 \\
\hline & & $\mu_{\hat{\tau}}$ & 40.27 & 40.04 & 40.41 & 40.24 & 40.11 & 40.07 & 40.21 & 40.02 & 39.95 \\
\hline & & $\sigma_{\hat{\tau}}$ & 4.00 & 4.50 & 5.61 & 4.05 & 3.54 & 4.00 & 3.88 & 4.88 & 3.96 \\
\hline & & $\bar{V}_{l r t}$ & 0.55 & 0.52 & 0.60 & 0.56 & 0.52 & 0.51 & 0.48 & 0.55 & 0.55 \\
\hline & & $\bar{M}_{l r t}$ & 14.30 & 14.53 & 13.59 & 13.71 & 13.71 & 14.29 & 14.89 & 13.88 & 14.81 \\
\hline & \multirow[t]{5}{*}{30} & $p d$ & 1.00 & 1.00 & 1.00 & 1.00 & 1.00 & 1.00 & 1.00 & 1.00 & 1.00 \\
\hline & & $\mu_{\hat{\tau}}$ & 40.08 & 40.10 & 40.03 & 40.05 & 40.05 & 40.08 & 40.09 & 40.02 & 40.13 \\
\hline & & $\sigma_{\hat{\tau}}$ & 1.67 & 1.70 & 1.88 & 1.87 & 1.87 & 1.67 & 1.83 & 2.09 & 2.00 \\
\hline & & $\bar{V}_{l r t}$ & 0.46 & 0.45 & 0.46 & 0.44 & 0.44 & 0.44 & 0.48 & 0.51 & 0.44 \\
\hline & & $\bar{M}_{l r t}$ & 25.39 & 25.63 & 24.57 & 24.26 & 24.26 & 26.37 & 26.30 & 24.31 & 26.72 \\
\hline & \multirow[t]{5}{*}{40} & $p d$ & 1.00 & 1.00 & 1.00 & 1.00 & 1.00 & 1.00 & 1.00 & 1.00 & 1.00 \\
\hline & & $\mu_{\hat{\tau}}$ & 39.94 & 40.02 & 40.01 & 40.01 & 40.02 & 40.03 & 39.96 & 40.02 & 40.00 \\
\hline & & $\sigma_{\hat{\tau}}$ & 0.95 & 0.99 & 1.08 & 0.92 & 0.89 & 0.94 & 0.91 & 0.92 & 0.80 \\
\hline & & $\bar{V}_{l r t}$ & 0.45 & 0.45 & 0.45 & 0.48 & 0.45 & 0.45 & 0.45 & 0.43 & 0.47 \\
\hline & & $\bar{M}_{l r t}$ & 38.19 & 38.33 & 36.36 & 36.56 & 39.93 & 39.32 & 39.02 & 36.46 & 38.99 \\
\hline
\end{tabular}

been discussed. However, in practice, several change points usually occur in the supply chain network and how to design control charts for monitoring this case deserves further research. Furthermore, performance of the change-point control chart should be evaluated under different shifts, e.g., in drifts and outliers, as well. This may also deserve further research.

\section{Acknowledge}

This work has been supported by the Natural Science Foundation of China (No. 71801049, 71802110), the Humanities and Social Sciences Foundation of MOE (Ministry of Education in China) (No. 17YJC630231,17YJC630043), and the FAFU 
Table 8. Performance with misspecified values of the parameters $A_{k^{*}}$ and $C_{k^{*}}(\tau=30, \xi=2)$.

\begin{tabular}{|c|c|c|c|c|c|c|c|c|c|c|c|}
\hline$k^{*}$ & $\delta$ & Indicator & True & $a$ & $b$ & $c$ & $d$ & $e$ & $f$ & $g$ & $h$ \\
\hline \multirow[t]{15}{*}{2} & 20 & $p d$ & 0.99 & 0.99 & 0.97 & 0.97 & 0.99 & 0.99 & 0.99 & 0.97 & 0.99 \\
\hline & & $\mu_{\hat{\tau}}$ & 29.95 & 29.98 & 29.87 & 29.96 & 29.97 & 28.91 & 29.93 & 29.72 & 30.00 \\
\hline & & $\sigma_{\hat{\tau}}$ & 1.89 & 2.05 & 2.26 & 2.30 & 2.04 & 1.96 & 1.89 & 2.71 & 1.68 \\
\hline & & $\bar{V}_{l r t}$ & 0.54 & 0.54 & 0.60 & 0.54 & 0.54 & 0.52 & 0.56 & 0.62 & 0.52 \\
\hline & & $\bar{M}_{l r t}$ & 13.89 & 13.81 & 12.54 & 12.64 & 13.81 & 14.65 & 14.72 & 12.69 & 14.47 \\
\hline & 30 & $p d$ & 1.00 & 1.00 & 1.00 & 1.00 & 1.00 & 1.00 & 1.00 & 1.00 & 1.00 \\
\hline & & $\mu_{\hat{\tau}}$ & 29.95 & 30.01 & 29.98 & 29.98 & 30.01 & 29.95 & 29.98 & 29.98 & 29.96 \\
\hline & & $\sigma_{\hat{\tau}}$ & 0.83 & 0.71 & 0.80 & 0.91 & 0.79 & 0.74 & 0.68 & 0.82 & 0.71 \\
\hline & & $\bar{V}_{l r t}$ & 0.49 & 0.49 & 0.53 & 0.50 & 0.45 & 0.54 & 0.48 & 0.53 & 0.48 \\
\hline & & $\bar{M}_{l r t}$ & 23.17 & 22.94 & 20.85 & 20.88 & 23.15 & 24.39 & 29.55 & 21.14 & 24.60 \\
\hline & 40 & $p d$ & 1.00 & 1.00 & 1.00 & 1.00 & 1.00 & 1.00 & 1.00 & 1.00 & 1.00 \\
\hline & & $\mu_{\hat{\tau}}$ & 30.00 & 30.00 & 30.00 & 29.98 & 29.99 & 30.00 & 30.02 & 29.98 & 30.00 \\
\hline & & $\sigma_{\hat{\tau}}$ & 0.32 & 0.33 & 0.47 & 0.44 & 0.35 & 0.31 & 0.29 & 0.49 & 0.32 \\
\hline & & $\bar{V}_{l r t}$ & 0.48 & 0.47 & 0.49 & 0.46 & 0.48 & 0.45 & 0.49 & 0.53 & 0.51 \\
\hline & & $\bar{M}_{l r t}$ & 31.89 & 32.05 & 29.27 & 29.29 & 31.81 & 33.56 & 33.42 & 29.34 & 33.31 \\
\hline \multirow[t]{15}{*}{$1,3,5$} & 20 & $p d$ & 0.99 & 0.99 & 0.99 & 0.99 & 0.99 & 0.99 & 0.99 & 0.99 & 0.99 \\
\hline & & $\mu_{\hat{\tau}}$ & 29.95 & 29.92 & 29.92 & 29.96 & 29.87 & 29.86 & 29.89 & 29.84 & 29.91 \\
\hline & & $\sigma_{\hat{\tau}}$ & 1.89 & 1.94 & 1.93 & 1.86 & 2.03 & 2.10 & 2.00 & 2.21 & 2.10 \\
\hline & & $\bar{V}_{l r t}$ & 0.54 & 0.49 & 0.53 & 0.50 & 0.53 & 0.59 & 0.54 & 0.52 & 0.52 \\
\hline & & $\bar{M}_{l r t}$ & 13.89 & 13.69 & 13.74 & 13.84 & 13.89 & 13.85 & 13.69 & 13.95 & 13.86 \\
\hline & 30 & $p d$ & 1.00 & 1.00 & 1.00 & 1.00 & 1.00 & 1.00 & 1.00 & 1.00 & 1.00 \\
\hline & & $\mu_{\hat{\tau}}$ & 29.95 & 29.92 & 29.99 & 29.95 & 29.98 & 29.96 & 29.94 & 29.98 & 29.98 \\
\hline & & $\sigma_{\hat{\tau}}$ & 0.83 & 0.76 & 0.79 & 0.72 & 0.64 & 0.73 & 0.84 & 0.76 & 0.81 \\
\hline & & $\bar{V}_{l r t}$ & 0.49 & 0.49 & 0.49 & 0.51 & 0.48 & 0.52 & 0.48 & 0.52 & 0.50 \\
\hline & & $\bar{M}_{l r t}$ & 23.17 & 23.14 & 23.07 & 23.21 & 23.28 & 23.09 & 23.12 & 23.03 & 22.90 \\
\hline & 40 & $p d$ & 1.00 & 1.00 & 1.00 & 1.00 & 1.00 & 1.00 & 1.00 & 1.00 & 1.00 \\
\hline & & $\mu_{\hat{\tau}}$ & 30.00 & 29.98 & 29.99 & 29.99 & 30.00 & 29.99 & 30.01 & 29.99 & 29.98 \\
\hline & & $\sigma_{\hat{\tau}}$ & 0.32 & 0.36 & 0.42 & 0.32 & 0.36 & 0.39 & 0.31 & 0.37 & 0.35 \\
\hline & & $\bar{V}_{l r t}$ & 0.48 & 0.45 & 0.53 & 0.47 & 0.50 & 0.47 & 0.48 & 0.48 & 0.46 \\
\hline & & $\bar{M}_{l r t}$ & 31.89 & 31.85 & 31.81 & 31.95 & 31.96 & 31.86 & 31.67 & 31.74 & 31.76 \\
\hline
\end{tabular}

Foundation for Distinguished Young Scholars (No. xjq201736).

\section{References}

1. Xu, X., Thuong, T.X., Kim, H.S., and You, S.S. "Optimising supply chain management using robust control synthesis", International Journal of Logistics Economics \& Globalisation, 7(3), pp. 277-291 (2018).

2. Gitinavard, H., Shirazi, M., and Ghodsypour, S. "A bi-objective multi-echelon supply chain model with Pareto optimal points evaluation for perishable products under uncertainty", Scientia Iranica, 26(5), pp. 2952-2970 (2019). DOI: 10.24200/SCI.2018.5047.1060

3. Zhong, J., Ma, Y., and Tu, P. "Supply chain qual- ity management: An empirical study", International Journal of Contemporary Hospitality Management, 28(11), pp. 2446-2472 (2016).

4. Heydari, J. "Lead time variation control using reliable shipment equipment: An incentive scheme for supply chain coordination", Transportation Research Part E: Logistics and Transportation Review, 63(3), pp. 44-58 (2014).

5. Montgomery, D.C. "A discussion on statistically-based process monitoring and control", Journal of Quality Technology, 29(2), pp. 157-162 (1997).

6. Woodall, W.H., Tsui, K.L., and Tucker, G.R. "A review of statistical and fuzzy quality control charts based on categorical data", Frontiers in Statistical 
Quality Control, Physica-Verlag HD, 5, pp. 83-89 (2012).

7. Wang, F.S., "Study on supply chain decision and monitoring based on response time", Doctoral Dissertation of Huazhong University of Science and Technology (2006).

8. Chen, K., Shaw, Y., and Yi, C. "Applying back propagation network to cold chain temperature monitoring", Advanced Engineering Informatics, 25(1), pp. 11-22 (2012).

9. Wang, S.S., Teresa, W., Shaojen, W., and John, F. "A control chart based approach to monitoring supply network dynamics using Kalman filtering", International Journal of Production Research, 50(11), pp. 3137-3151(2012).

10. Faraz, A., Heuchenne, C., Saniga, E., and Foster E. "Monitoring delivery chains using multivariate control charts", European Journal of Operational Research, 228(1), pp. 282-289 (2013).

11. Lu, S.L. and Tsai, C.F. "A nonparametric GA-GWMA sign chart for green SCM optimization", IEEE International Conference on e-Business Engineering, pp. 504-508 (2013).

12. Zhong, J., Ma, Y., and Tu, P. "Integration of SPC and performance maintenance for supply chain system", International Journal of Production Research, 54(19), pp. 5932-5945 (2016).

13. Tsung, F., Li, Y.T., and Jin, M. "Statistical process control for multistage manufacturing and service operations: a review and some extensions", International Journal of Services Operations and Informatics, 3(2), pp. 191-204 (2008).

14. Zolfaghari, S. and Amiri, A. "Monitoring multivariateattribute quality characteristics in two stage processes using discriminant analysis based control charts", Scientia Iranica, Transactions E: Industrial Engineering, 23(2), pp. $757-767$ (2016).

15. Amiri, A. and Zolfaghari, S. "Estimation of change point for multistage processes subject to step change and linear trend", International Journal of Reliability, Quality and Safety Engineering, 23(2), p. 1650007 (2016).

16. Assareh, H., Noorossana, R., Mohammadi, M., and Mengersen K. "Bayesian multiple change-point estimation of Poisson rates in control charts", Scientia Iranica, 23(1), pp. 316-329 (2016).

17. Shang, Y.F., Tsung, F., and Zou, C.L. "Statistical process control for multistage process with binary outputs", IIE Transactions, 45(9), pp. 1008-1023 (2013).

18. Pirhooshyaran, M. and Niaki, S.T.A.A. "Doublemax MEWMA scheme for simultaneous monitoring and fault isolation of multivariate multistage autocorrelated processes based on novel reduced-dimension statistics", Journal of Process Control, 29, pp. 11-22 (2015).

19. Bazdar, A., Reza, B.K., and Niaki, S.T.A. "Fault diagnosis within multistage machining processes using linear discriminant analysis: a case study in automotive industry", Quality Technology \& Quantitative Management, 14(2), pp. 129-141 (2017).

20. Zou, N. and Li, J. "Modeling and change detection of dynamic network data by a network state space model", IISE Transactions, 49(1), pp. 45-57 (2017).

21. Jin, M., Li, Y., and Tsung, F. "Chart allocation strategy for serial-parallel multistage manufacturing process", IIE Transactions, $42(8)$, pp. 577-588 (2010).

22. Sullivan, J.H. and Woodall, W.H. "A control chart for preliminary analysis of individual observations", Journal of Quality Technology, 28(3), pp. 265-278 (1996).

23. Zou, C.L., Tsung, F., and Liu, Y. "A change point approach for phase I analysis in multistage process", Technometrics, 50(3), pp. 344-356 (2008).

\section{Biographies}

Jianlan Zhong is currently an Associated Professor in the College of Management and the College of Tourism, Fujian Agriculture and Forestry University, P.R. China. Her main area of interest is quality control in supply chain context.

XueLong $\mathbf{H u}$ is currently an Assistant Professor in the School of Management, Nanjing University of Posts and Telecommunications, P.R. China. His main area of interest is new statistical quality control techniques.

Yaqing Yang is an MSc student in the College of Economics and Management, Nanjing University of Science and Technology, P.R. China. Her main area of interest is quality control.

Yi Liu (Paul) Tu is a Professor in the Department of Mechanical and Manufacturing Engineering, University of Calgary, Canada. He is Chairman of board at CIM Systems Ltd., Calgary, Alberta, Canada, and Zi Jin Scholar Chair Professor in the College of Economics and Management, Nanjing University of Science and Technology, P.R. China. He is an active researcher in OKP (One-of-a-Kind Production) product design and manufacture as well as ultra-fast laser micro-machining technology. 